\title{
THE ABSORPTION OF SOUND AT OBLIQUE ANGLES OF INCIDENCE
}

\author{
By P. R. Heyl, V. L. Chrisler, and W. F. Snyder
}

ABSTRACT

The absorption of sound at oblique angles of incidence has up to the present been purely a matter of theory, no experimental work having been published on the subject. Theoretical discussions have been given, which appear to be in error in an essential point, namely, the overlooking of the probable existence of rotational motion in the region of absorption.

This paper describes experiments made to investigate this question. It is found that the absorption varies with the angle of incidence, but not according to the law which has been deduced from purely theoretical considerations.

As a consequence of the experiments described in this paper the conclusion has been reached that the tube method of measuring absorption is limited in its application to relative comparison of samples of similar nature, and that for absolute values of absorption the reverberation method is the only trustworthy one.

\section{CONTENTS}

I. Origin of the question

Page

II. Theoretical discussion 289

III. Experimental results

IV. Discussion of results

V. Conclusions

295

\section{ORIGIN OF THE QUESTION}

Two methods are in general use for determining the coefficient of absorption of sound of a material - the tube method ${ }^{1}$ and the reverberation method. ${ }^{2}$ The results obtained by these two methods have frequently been discordant to an extent that can not be accounted for as experimental error. For instance, Celotex BB at 512 cycles may show by the tube method an absorption coefficient of 0.31 , and in the reverberation room 0.61. With Balsam Wool the results may differ still more widely, 0.16 and 0.55 . This discrepancy is found with most substances, though exceptions occur, especially with materials of low absorption, such as glass.

It has been suggested that such differences might arise from the fact that in the tube method as usually applied the incidence of sound upon the test plate is normal, while in the reverberation room the sound reaches the sample at all angles.

A considerable element of uncertainty has always attached itself to measurements of sound absorption. There have been but few work-

1 J. Tuma, Akad. d. Wiss. Wien, Ber., 3 (2a), p. 402; 1902. H. O. Taylor, Phys. Rev., 2, p. $270 ; 1913$. E. A. Eckhardt and V. L. Chrisler, Transmission and Absorption of Sound by Sonne Building Materials, B. S. Sci. Paper No. 526; 1926.

Sound by Sabine, Collected Papers, Harvard Univ. Press; 1922. F. R. Watson, The Absorption of Sound by Materials, Bulletin No. 172, Exp. Sta., Univ, of Illinois; 1927. 
ers in the subject, and these have sometimes failed to agree, while at other times the concordance of results has been encouragingly satisfactory. Of these observers, some have used one method and some the other, and until recently no one laboratory has been equipped to make comparative tests by both methods and by the same observers.

With the completion of the new reverberation chamber at the Bureau of Standards such an opportunity offered itself. The results of the work were at first disappointing. Instead of eliminating the discrepancies they were confirmed. Analysis of the results. however, has led to certain conclusions of interest.

\section{THEORETICAL DISCUSSION}

Paris ${ }^{3}$ has published a mathematical discussion of the absorption of sound at any angle of incidence in which he gives a formula which, applied to a material of coefficient 0.28 at normal incidence, indicates an increase of about 25 per cent at $45^{\circ}$ and of 50 per cent at $60^{\circ}$.

This paper by Paris was the outgrowth of an earlier paper by the late Lord Rayleigh." Both of these authors treat of sound waves in the classical way, representing the motion by the usual differential equation in which the dependent variable is the velocity potential $\phi$. Paris (p. 411) gives expressions for $\phi$ in the incident and reflected beams and lays down a condition which $\phi$ must satisfy at the reflecting surface, from which condition he derives an expression for the absorption.

Now sound absorption, when present to any considerable degree, is usually a consequence of porosity. In the act of reflection the air immediately in contact with the surface is forced in and out of capillary channels in the material, encountering frictional resistance which converts a portion of the sound energy into heat. This mechanism of absorption was recognized and clearly stated by Rayleigh. ${ }^{5}$

But friction is particularly prone to set up rotational motion in fluids, and in a region of rotational motion there can be no velocity potential.

Such rotational motion will probably disappear again because of viscosity at a very short distance from the reflecting surface. It would, therefore, appear that over a thin layer of air close to the absorbing surface the usual differential equation for sound motion in air is not valid.

Even with glass, which has no sensible pores, there is an appreciable amount of absorption of sound which must arise from friction of some kind, probably of a sliding nature; and to the extent to which friction plays a part in reflection conclusions based upon the existence of a velocity potential must be uncertain.

It must be admitted that this criticism applies equally well to such a theory of the tube method as is given in Bureau of Standards Scientific Paper No. 526. ${ }^{6}$ Taylor's derivation, ${ }^{6}$ however, seems free from this theoretical objection, and yet his formula for the absorption agrees perfectly with that obtained by Eckhardt and Chrisler.

\footnotetext{
E. T. Paris, Proc. Roy. Soc., A, 115, p. 407; 1927

Rayleigh, Phil. Mag., 39, p. 225; 1920.

- Seo footnote 4.

See footnote 1, p. 289.
} 
Where theory stands on such uncertain ground recourse must be had to experiment, and up to the present time no experimental work on this subject has been published.

\section{EXPERIMENTAL RESULTS}

Without reproducing a mass of detail it may be said that the result of experiments on a great variety of materials by both the tube and the reverberation methods by the same observers has been to confirm the difference in results usually found by the two methods, the tube method giving the lower results. It has been noticed, however, that at high frequencies these differences may disappear. Table 1 gives results for two materials which illustrate this point well.

TABLE 1.-Comparison of absorption coefficients at different frequencies

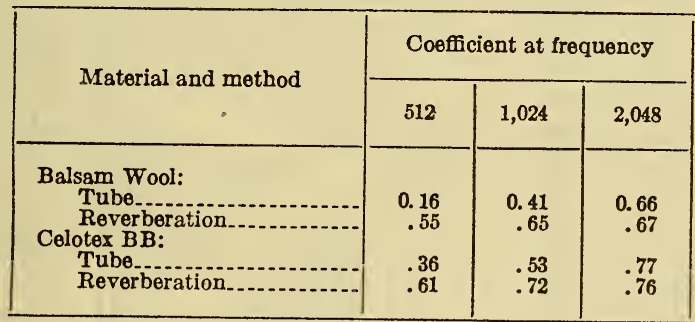

Results of this nature suggest that there is something in acoustics analogous to what in atomic physics is called the "correspondence principle." The results correspond well at high frequencies but not at low ones. In view of this agreement at high frequencies the reason for supposing the absorption to vary with the angle of incidence is somewhat weakened. Following the tradition as set by the work of W. C. Sabine, measurements of coefficient of absorption were at first made mostly at 512 cycles, as representing a fair average of the different frequencies with which acoustic work has to deal. Had it happened that a frequency of 2,000 or more had represented this average it is doubtful whether the question of variation of cofficient with angle of incidence would then have arisen.

The difference at low frequencies may be due in part to the fact that with large samples, such as are used in the reverberation room, there may occur structural vibration which is not present in the much smaller samples used in the tube method. Such vibration, with accompanying dissipation of energy, would be more in evidence at low frequencies; but there is also another factor which may enter into the case, and which will be mentioned later.

For a more direct investigation of the question of absorption at an oblique angle of incidence a special form of apparatus was devised, shown in Figure 1. This was a modification of the usual form of tube apparatus as used for normal incidence, and was built in two forms, for angles of incidence of $45^{\circ}$ and $60^{\circ}$, respectively. The form illustrated in Figure 1 is that for $45^{\circ}$. The $45^{\circ}$ apparatus as actually set up is shown in Figure 2. 
The sound produced by a loud speaker passed by two equal paths to the ends of the absorption apparatus. This consisted of an elbow of large brass tubing, open at the bend, to which opening the test plate was applied. Stationary waves were set up in each leg of the absorption apparatus, and the measurements involved were the same as with the usual type at normal incidence, except for the fact that observations at oblique incidence could not be taken close to the plate.

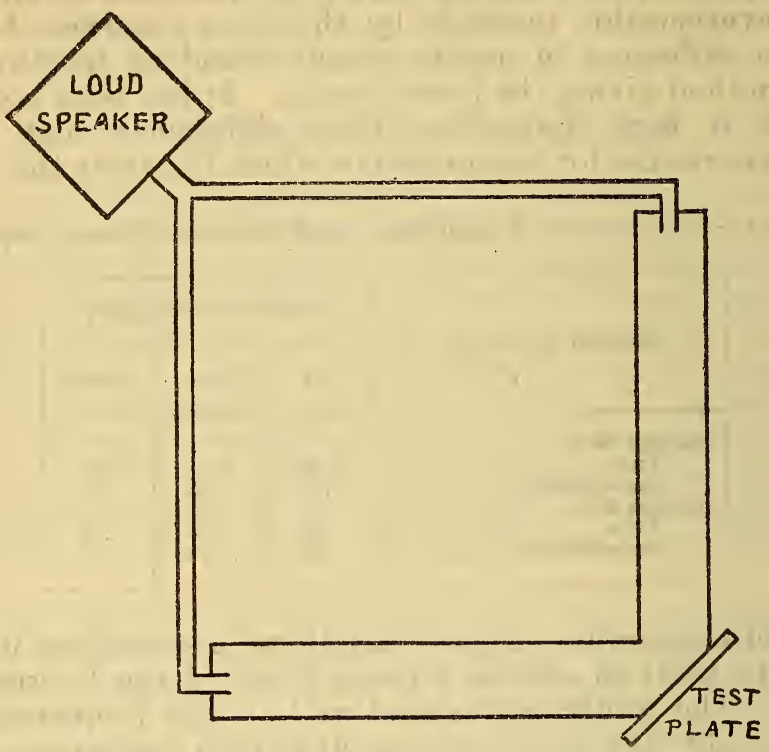

FigdRe 1.-Tube apparatus for $45^{\circ}$ incidence.

With this apparatus measurements were made at angles of incidence of $45^{\circ}$ and $60^{\circ}$ on glass, Masonite, Celotex, and Akoustolith, and the results were compared with figures obtained for these materials at normal incidence with the usual type of apparatus. Table 2 gives the results thus obtained.

TABLE 2.-Coefficients of absorption by the tube method at different angles of incidence

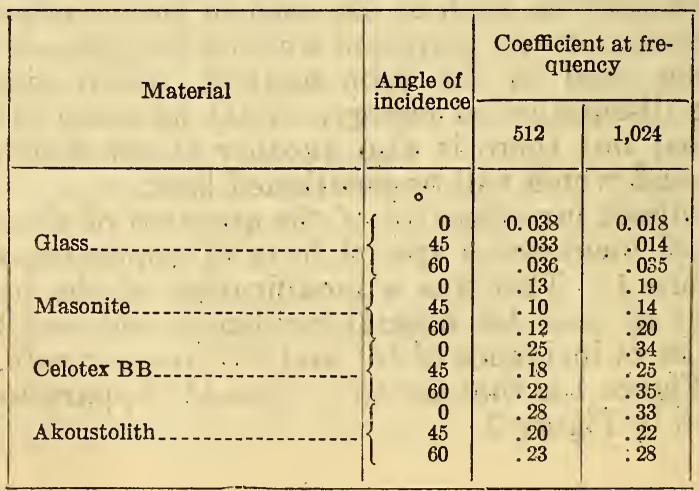




\section{B. S. Journal of Research, RPI49}

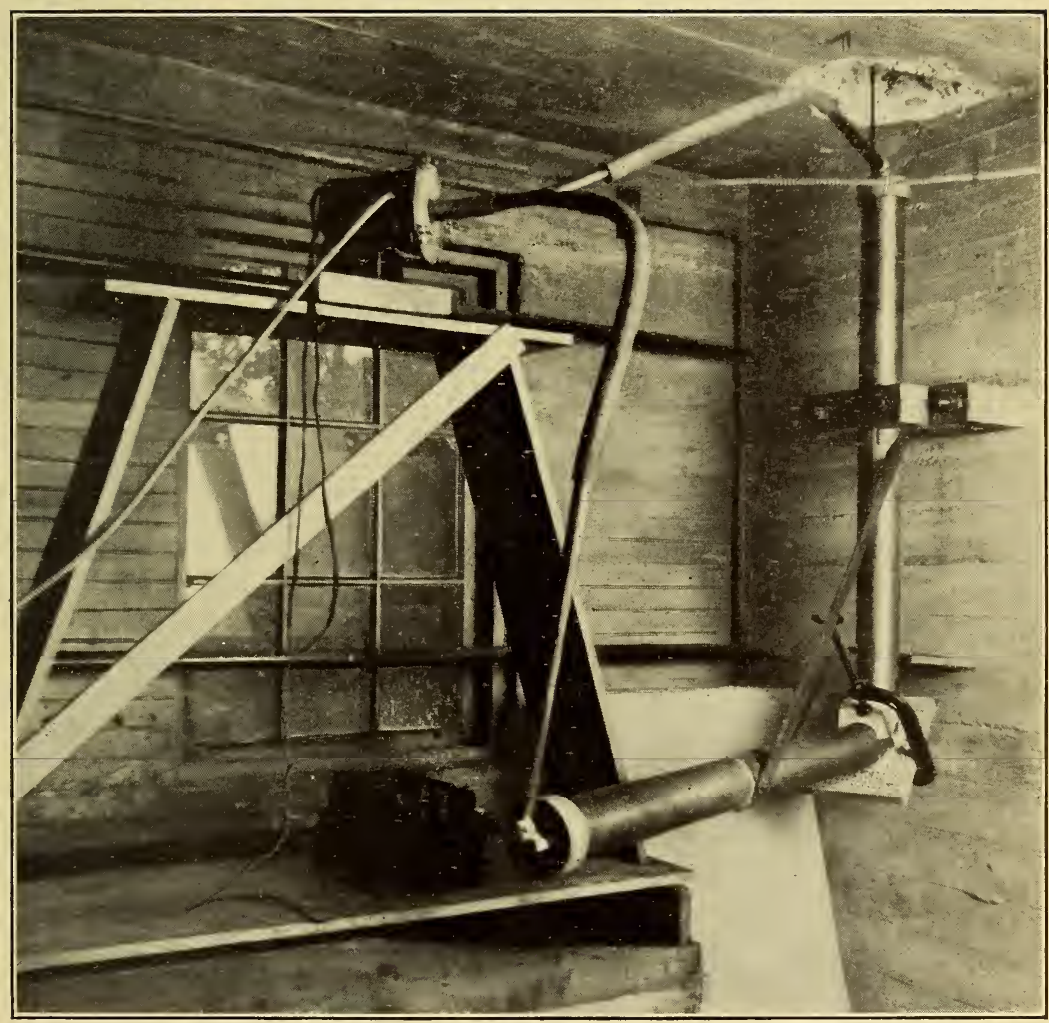

Figure 2.-Arrangement of apparatus for $45^{\circ}$ incidence 
Of the materials listed in Table 2, Akoustolith and Celotex (at 512 cycles) correspond closely to the supposed material of 0.28 absorption at normal incidence to which Paris applied his formula, but the experimental results for these materials at oblique angles are quite different from those indicated by Paris's calculation. Instead of increasing, the absorption decreases at both $45^{\circ}$ and $60^{\circ}$.

This decrease at $45^{\circ}$ is in evidence for all materials given in the table and at both frequencies. There appears to be a recovery at $60^{\circ}$, partial or complete, but the only certain case of increase at this angle (that of glass) is about 100 per cent instead of 50 per cent.

These results, as far as they go, indicate that while the angle of incidence may have an effect upon the coefficient of absorption, it is not that indicated by the formula of Paris.

In experiments such as these it is not possible to have all points of the test plate simultaneously in a node, as may be the case at normal incidence. The question of absorption at oblique incidence with every point a nodal point is rather an academic one, as such a condition is impossible of realization. However, in these experiments care was taken to adjust the apparatus so that (at least in theory) the short diameter of the elliptical test surface exposed to the sound should be a nodal line, of maximum pressure change, with a symmetrical diminution in pressure on either side. The average pressure over the test plate was thus the greatest attainable for that angle of incidence, though of course less than the theoretical pressure at normal incidence; and since absorption in capillaries is doubtless dependent upon pressure, a lower value of the absorption might be expected in all cases of oblique incidence. That this is the case at $45^{\circ}$ but not at $60^{\circ}$ is rather puzzling.

To check experimentally this suggested relation between absorption and pressure a new sample of Masonite was applied to the $45^{\circ}$ apparatus. Adjusting for maximum pressure along a short diameter, readings were obtained. The adjustment was then changed so as to bring the nodal line near one end of the test plate, with a consequent lowering of the average pressure over the whole plate, and readings were again taken. Table 3 gives the coefficients of absorption thus obtained.

TABLE 3.-Coefficients of absorption at different pressures

[Masonite, tube method, $45^{\circ}$ incidence]

\begin{tabular}{|c|c|c|}
\hline \multirow{2}{*}{ Distribution of pressure } & \multicolumn{2}{|c|}{$\begin{array}{c}\text { Coefficient at } \\
\text { frequency }\end{array}$} \\
\cline { 2 - 3 } & 512 & 1,024 \\
\hline & $\begin{array}{r}0.10 \\
.06\end{array}$ & $\begin{array}{r}0.18 \\
.11\end{array}$ \\
\hline
\end{tabular}

It will be noticed that the coefficients of absorption obtained in the symmetrical case agree quite well with those obtained under similar conditions as given in Table 2, but that with the unsymmetrical distribution and consequent lower average pressure the absorption falls off considerably. 


\section{DISCUSSION OF RESULTS}

We may briefly summarize the experimental facts presented.

1. The coefficient of absorption may vary with the fluctuation of pressure developed at the absorbing material while the intensity of the incident sound remains constant. (Table 3.)

2. The coefficient of absorption may vary with the angle of incidence, but not according to the formula of Paris. (Table 2.)

3 . The coefficient of absorption of most materials increases with the frequency, at least to about 2,000 cycles per second.

4. The coefficient of absorption usually measures higher in the reverberation chamber than by the tube method. This discrepancy disappears at high frequencies. (Table 1.)

It appears likely that the first of these facts is the underlying cause of the others, for reasons which we will now set forth.

In order to understand the reason for a difference in coefficient of absorption in the cases of normal and oblique incidence we must examine the pressure conditions produced in each case at the absorbing surface. This pressure arises from the development of a region of alternate condensation and rarefaction at a certain place on the surface. Since a sound wave is not a convection current, and since fluid pressure is equal in all directions, the maximum pressure at any point of the surface will be independent of the angle of incidence.

Not so with the average pressure over the test plate. This may vary considerably with the angle of incidence. With a plane wave normally incident upon a plane surface it may be that every point of the surface will be simultaneously in a node. This is impossible with an obliquely incident wave, which can give only a nodal line of maximum pressure change with a diminishing pressure change and increasing motion on either side; and the greater the angle of incidence the more rapidly will the pressure change fall off on either side of the nodal line. The average pressure change, and, consequently, the coefficient of absorption, should, therefore, diminish steadily with increasing obliquity of incidence.

This is opposed to Paris's theoretical conclusion and, what is more important, partly in disagreement with the results on absorption in Table 2, which exhibit a falling off at $45^{\circ}$ and a recovery at $60^{\circ}$.

In explanation of this contradiction with experiment it may be pointed out that it is impossible to realize a pure case of either normal or oblique incidence by the tube method. This fact was not realized when the apparatus for measurements at oblique incidence was designed, and was recognized only after a study of the results obtained. The sound wave is conducted to the upper end of the brass tube by a smaller pipe, and leaves the orifice of this pipe not as a plane wave, but rather as a spherical one. Consequently, there must be reflection from the walls of the tube. These walls have but little absorption, and the reflection is repeated all the way down the tube. There may be also a distortion of an otherwise plane wave due to viscous drag along the sides of the tube. Though a part of the original wave may reach the test plate at the bottom of the tube in a nearly plane condition, there is to be considered the interference arising from that part of the wave which has reached the bottom by the zigzag path of 
reflection. We must, therefore, expect to find the pressure pattern over the test plate considerably broken up and the average pressure altered to an unknown extent.

It is also impossible to realize a pure case of incidence at a single angle in the reverberation chamber. Here the absorbing surface is exposed to sound waves which, because of the distance of the source, may be approximately plane, but which meet the absorbing surface at various angles and in different phases, determined by the size and shape of the chamber and the relative positions of the source and the absorbing surface. In reverberation work an attempt is made to break up and subdivide the interference pattern in the chamber as finely as possible. Of this pattern the distribution of pressure over the absorbing surface is, at any instant of time, a cross-sectional representation. Were it possible to carry this subdivision to the limit the pressure at any given instant of time would be constant over the whole absorbing surface, and the sound waves might be described as perfectly shuffled. In practice such a condition is unattainable. The distribution of pressure will always be one of points of maximum and equal pressure change separated by regions of less pressure, with a consequent average less than the theoretical maximum for normal incidence. But the shorter the wave length the more closely will these maximum points be spaced, and the average pressure over the surface will rise with the frequency. This may be the chief factor in the explanation of the increase in absorption with frequency characteristic of all materials, and of the "correspondence principle" in Table 1.

But why should results obtained in the reverberation chamber come out higher than those obtained by the tube method arranged for normal incidence? The answer must be that we do not get pure normal incidence, but a pressure pattern broken up by reflection from the sides of the tube. And since the coefficient under these circumstances comes out less than by the reverberation method, the only conclusion is that the average pressure in the tube must be lower than that in the reverberation chamber. In the absence of any other explanation the above is put forward tentatively.

Though no definite angle of incidence can be obtained by the reverberation method, the average condition which prevails, with such subdivision of the interference pattern as is usually possible, is one which should be capable of approximate reproduction in any other reverberation chamber and one which corresponds quite well to conditions which prevail in any practical construction to which the tested material is to be applied. The utility of the tube method is confined to cases of rapid comparison of small samples on a relative basis, and this method should not be trusted to give absolute values except at high frequencies.

\section{CONCLUSIONS}

Because of the probability of rotational motion being set up in a region where sound absorption is taking place, it is unsafe to apply to such a region mathematical analysis involving the assumption of the existence of a velocity potential. Theoretical conclusions based upon such reasoning are invalid. 
Physical considerations suggest that absorption should be a maximum at normal incidence. This is confirmed to a certain extent by the results in Table 3 . It is impossible, however, to realize a welldefined case of incidence confined to any angle either by the tube method or in the reverberation chamber.

In the practical measurement of absorption coefficients the reverberation method should be the only one trusted for absolute values of the coefficient, suitable for use under conditions that prevail in actual construction.

WASHINGTON, September 10, 1929. 